Check for updates

Cite this: RSC Adv., 2020, 10, 2060

Received 3rd November 2019

Accepted 21st December 2019

DOI: $10.1039 / \mathrm{c} 9 \mathrm{ra09079g}$

rsc.li/rsc-advances

\section{The effect of magnetic field pretreatment on the corrosion behavior of carbon steel in static seawater}

\author{
Shuanzhu Zhao, ${ }^{a}$ Yaxin Wang, ${ }^{\text {b }}$ Yunxiu Zhao, ${ }^{d}$ Xiaotong Sun, ${ }^{b}$ Huijuan Zhang, ${ }^{\text {a }}$ \\ Hong-Guang Piao, (D) *ad Yujiao Zhang ${ }^{\mathrm{b}}$ and Yanliang Huang ${ }^{\mathrm{c}}$
}

\begin{abstract}
The corrosion behavior of carbon steel pretreated with a magnetic field before electrochemical testing was investigated in static seawater using electrochemical methods in the absence of an external magnetic field. The shift in corrosion potential was more significant with increasing pretreating magnetic field strength, and the corrosion current density also decreased. This implies that the carbon steel corrosion was inhibited. The main reason for this inhibition is that the magnetic field affects the formation of intermediate products on the carbon steel surface by both charge transfer and magnetic ion adsorption. The magnetic field pretreatment will likely offer a new approach for marine anti-corrosion technology.
\end{abstract}

\section{Introduction}

Seawater is a complex natural equilibrium system with high salinity, electrical conductivity, and biological activity. ${ }^{\mathbf{1}}$ As such, seawater is a highly corrosive electrolyte solution with a high concentration of $\mathrm{Cl}^{-}$(ref. 2) and microorganisms ${ }^{3}$ that can impact metal corrosion. Anti-corrosion coating ${ }^{4-6}$ and cathodic protection $^{7}$ are generally used to solve corrosion problems in carbon steel. However, the environmental-pollution effects of coal-tar in anti-corrosion coatings are inevitable, ${ }^{8}$ and galvanic anodes cannot economically deliver currents to provide complete protection. $^{7}$

Cleaner and more efficient methods are needed to solve metal corrosion problems in marine applications. Magnetic fields are promising in the field of anti-corrosion technologies because they can modulate the activity of corrosive microorganisms and are environmentally friendly. ${ }^{9-12}$

Carbon steel is widely used in engineering for its high cost performance, ${ }^{\mathbf{1 3}}$ but is still often corroded by seawater. Therefore, the corrosion behavior of carbon steel in seawater has attracted much attention in the marine resource development process. ${ }^{\mathbf{1 , 1 4}}$ Concurrently, carbon steel is sensitive to magnetic fields, and the formation of pitting corrosion will be affected..$^{15-18}$ Therefore, it is important to study the corrosion

${ }^{a}$ Research Institute for Magnetoelectronics \& Weak Magnetic-field Detection, College of Science, China Three Gorges University, Yichang 443002, P. R. China. E-mail: hgpiao@ctgu.edu.cn; zhanghuijuan82@126.com

${ }^{b}$ College of Electrical Engineering \& New Energy, China Three Gorges University, Yichang 443002, P. R. China

${ }^{c}$ CAS Key Laboratory of Marine Environmental Corrosion and Bio-fouling, Institute of Oceanology, Chinese Academy of Sciences, Qingdao 266071, China

${ }^{d}$ Department of Physics, Chungbuk National University, Cheongju 28644, Republic of Korea behavior of carbon steels affected by magnetic fields in static seawater.

The electrochemical corrosion process of carbon steel under magnetic fields can be controlled by the Lorentz force effect during charge transfer. ${ }^{19}$ This is consistent with magnetohydrodynamics (MHD). The diffusion time of ions and the concentration polarization at the electrode surface decrease with the movement of charged particles under the action of the Lorentz force. This can enhance the transfer process in the corrosion solution..$^{20-23}$ The MHD arises from the interaction of the moving charged particles under a magnetic field. These interactions obey eqn (1). ${ }^{24-26}$

$$
\vec{F}_{\text {MHD }}=\vec{J} \times \vec{B} .
$$

here, $\vec{J}\left(\mathrm{~kg} \mathrm{~m}^{-2} \mathrm{~s}^{-1}\right)$ is the flux density of the moving charged particles, and $\vec{B}(\mathrm{~T})$ is the magnetic field. There is movement of both electrons and ions in the charge transfer process. The movement of electrons refers to the collision process between positive/negative ions and neutral atoms: these are a chemical change. The movement of ions (mass transfer) is driven by the chemical potential difference that can occur between two different phases or in multiple phases. It can even occur in a single phase if there is a concentration difference, i.e., a physical change. However, it remains controversial which process is the most dominated by the magnetic field: charge or mass. ${ }^{19,27,28}$ Therefore, it is very important to study the charge transfer process in the electrochemical corrosion process of carbon steel in seawater under magnetic fields.

In addition to the effect of Lorentz force, paramagnetic ions (such as $\mathrm{Fe}^{2+}, \mathrm{Co}^{2+}$, and $\mathrm{Ni}^{2+}$ ) are also affected by the magnetic gradient force under nonuniform magnetic field. The nonuniform distribution of magnetic field can be caused by the external magnetic field itself, magnetization of magnetic materials, or 
concentration gradients of paramagnetic ions. ${ }^{29,30}$ The influence of the gradient force on the corrosion behavior of carbon steel is different from the Lorentz force and cannot be ignored.

While many papers have discussed the influence of magnetic field on corrosion, most of them only discussed the real-time effect of external magnetic fields on the electrochemical corrosion process in various chemical solutions. This limits conclusions about the magnetic effect on the carbon steel corrosion process in oceans. Here, we considered the seawater solution to reflect the actual situation. The influence of the magnetic field on the corrosion behavior of carbon steel was studied by magnetic field pretreatment. This contributes to the application of magnetic fields in the ocean. We further consider the influence of magnetic gradient forces caused by the magnetization of carbon steel as a magnetic material. We further analyzed whether the charge transfer (chemical change) or the mass transfer (physical change) is more affected by the magnetic field. The results help improve the impact of magnetic field on the corrosion resistance of carbon steel.

\section{Experimental}

\subsection{Preparation of samples}

The A3-Q235 carbon steel (99.95\% purity, Yangzhou Xiangwei Machinery) was a standard corrosion test piece $(70 \times 10 \times 2$ $\mathrm{mm})$. All test pieces were washed with deionized water and ethanol after polishing with 2000 mesh sandpaper. The surface of the carbon steel test piece (sample) was partially encapsulated with $0.5 \mathrm{~mm}$ epoxy resin coating except for the effective reaction window of $10 \times 10 \mathrm{~mm}^{2}$. The epoxy resin coating is a mixture of epoxy resin and polyamide resin (as a curing agent) at a mass ratio of $1: 1$.

Seawater for electrochemical corrosion experiments were from salt from Huanyu Aquarium. This met the standard requirements for artificial ocean water ASTMD 1141-98: $30 \mathrm{~g} \mathrm{~L}^{-1}$ at $\mathrm{pH}$ 8.2. An electromagnet device (East Changing Technologies, P90-60) was used to generate the magnetic field for the sample pretreatment. Fig. 1 shows that the magnetic field was applied in parallel to the effective reaction window plane with strengths of $0 \mathrm{mT}$ (Case-1), $20 \mathrm{mT}$ (Case-2), and $40 \mathrm{mT}$ (Case-3) to pretreat three types of carbon steel. The three samples were pretreated under different magnetic field conditions in seawater for $1800 \mathrm{~s}$ before testing.

\subsection{Electrochemical experiments}

The potentiostatic polarization curve, potentiodynamic polarization curve, and electrochemical impedance spectroscopy were measured by using three-electrode Teflon cell method without an external magnetic field. ${ }^{31}$ The $30 \times 30 \times 0.3 \mathrm{~mm}^{3}$ platinum electrode was used as a counter electrode, and as the reference electrode was a saturated calomel electrode (SCE). The electrochemical analyzer (Shanghai Chenhua CHI760e) was used for potential control and current measurement. In the quasi-steady state measurement of the galvanostatic polarization and the potentiostatic polarization, the current density was less than $0.05 \mathrm{~A} \mathrm{~cm}^{-2}$, and the constant potential was $-0.2 \mathrm{~V}$ (vs. SCE).
These conditions were used to reduce the impact of electric field on the $\mathrm{pH}$ value of electrolyte solution (seawater) during electrochemical corrosion.

Potentiodynamic polarization curves were measured in the range of $-0.6 \mathrm{~V}$ to $-0.8 \mathrm{~V}$ ( $v s$. SCE) with a scan rate of $1 \mathrm{mV} \mathrm{s}^{-1}$ based on an open circuit potential at $1800 \mathrm{~s}$. The electrochemical impedance spectroscopy (EIS) data were recorded from $10^{5} \mathrm{~Hz}$ to $0.01 \mathrm{~Hz}$ with a $5 \mathrm{mV}$ sinusoidal perturbing signal at the open circuit potential. In addition, all experiments were repeated more than three times to ensure the accuracy of the results.

\subsection{Microstructure and composition characterization}

Scanning electron microscopy (SEM, JEOL JSM-7500F, Japan) was used to characterize the microstructure of the carbon steel corrosion surface. To prepare a clean SEM sample, the corrosion surface of pretreated samples under three cases were washed with alcohol and deionized water after soaking for $1800 \mathrm{~s}$ in seawater. The composition of the corrosion products on the surface of the carbon steels was characterized by X-ray powder diffraction (XRD, Rigaku Ultima IV, Japan) with a conventional $\mathrm{Cu}-\mathrm{K} \alpha$ radiation source. To determine the intermediate composition of three-cases samples, two XRD powder samples were prepared for the contrastive analysis. One was washed with only alcohol and deionized water after sample pretreatments and then naturally dried in air environment. The other one was artificially dried two hours at $70^{\circ} \mathrm{C}$ environment temperature with an electro-thermostatic blast oven (CIMO DHG-907385-III, China) after the sample was washed.

\section{Results and discussion}

\subsection{Galvanostatic polarization measurement}

To determine the effect of the magnetic field on the electrochemical corrosion potential $(E)$ of carbon steel, the pulsed magnetic field of $40 \mathrm{mT}$ strength and the $\sim 150 \mathrm{~s}$ pulse width was used along the direction parallel to the surface of the carbon steel $(B \perp E)$ in galvanostatic polarization measurements under different polarization current densities of $0.01 \mathrm{~A} \mathrm{~cm}^{-2}$, $0.015 \mathrm{~A} \mathrm{~cm}^{-2}, 0.02 \mathrm{~A} \mathrm{~cm}^{-2}$, and $0.025 \mathrm{~A} \mathrm{~cm}^{-2}$ as shown in Fig. 1(a). When the pulsed magnetic field was turned "ON", the corrosion potential of the carbon steel rapidly shifted cathodically. This means that the magnetic field may promote the corrosion behavior of carbon steel in seawater: This is due to the effect of the magnetic field on the mass-transfer behavior of carbon steel corrosion processes. ${ }^{32-34}$ The corrosion potential returned to its original level after the magnetic field was turned "OFF" though there was some deviation. The shifting value $(\Delta E)$ of the corrosion potential of the carbon steel changed with increasing polarization current density under the pulsed magnetic field of $40 \mathrm{mT}$. The $\Delta E$ is most significant at $0.02 \mathrm{~A}$ $\mathrm{cm}^{-2}$ as shown in Fig. 1(b), which means that the higher polarization current intensity of $0.025 \mathrm{~A} \mathrm{~cm}^{-2}$ may have triggered other electrochemical reactions that affected the corrosion potential of carbon steel, although the $\mathrm{pH}$ of seawater did not change significantly. During the magnetic field pretreatment of carbon steel, the applied direction of magnetic field was 

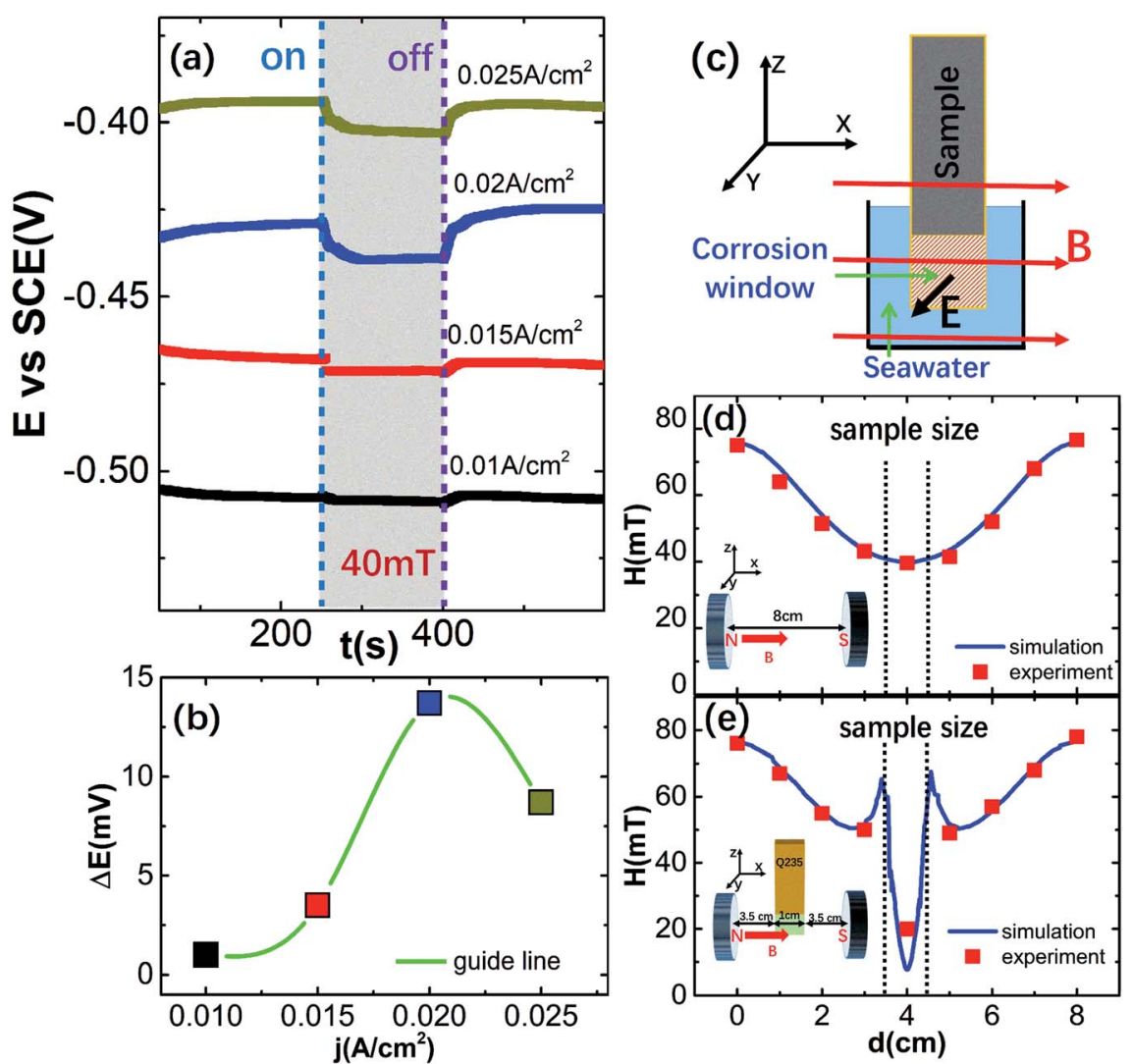

Fig. 1 (a) Galvanostatic polarization curves over time for carbon steel with changing the polarization current density under the pulsed magnetic field of $40 \mathrm{mT}$ strength and $150 \mathrm{~s}$ pulse width; (b) anodic shift $\Delta E$ under different polarization current density; (c) schematic diagram of experiment; (d) without carbon steel and (e) with carbon steel between the two magnetic poles, distributions of magnetic flux density.

parallel to the effective corroded surface of carbon steel (namely $B \perp E)$, and the carbon steel was located in the center position of two magnetic poles (Fig. 1(c)).

To understand the effect of the magnetic field on carbon steel pretreatment, the distribution of magnetic field between the two magnetic poles was analyzed first. As a reference for comparative analysis, the distribution of magnetic field along a central axis between two magnetic poles is depicted without the carbon steel by actual measurements and numerical finite element analysis. Fig. 1(d) shows that the magnetic field strength $(\sim 40 \mathrm{mT})$ is evenly distributed at the center between the two magnetic poles where the carbon steel is to be placed. Interestingly, the distribution of the magnetic field changes greatly (Fig. 1(e)) after the carbon steel is placed in the center position between the two magnetic poles.

Carbon steel is a magnetic material with high permeability and magnetization, and it inevitably forms a large magnetic gradient distribution near both edges of the carbon steel. This has also been verified by actual measurements and numerical finite element analysis (the data were collected from a center axis $1 \mathrm{~mm}$ above the surface of carbon steel). The magnetic field intensity at the both edges of the carbon steel could reach $50 \mathrm{mT}$ while those at the center surface of the carbon steel only reached $20.2 \mathrm{mT}$ from the experimental data. Therefore, the enhancing effect of the Lorentz force on the mass-transport processes will play an important role in the case of $B \perp E$ during the magnetic field pretreatment of carbon steel. ${ }^{35}$ Meanwhile, the suppressing and adsorptive effects of the magnetic gradient force affect convection of electrolyte solution and the iron ions $\left(\mathrm{Fe}^{2+}\right.$ and $\left.\mathrm{Fe}^{3+}\right)$, respectively. This occurs on the surface of the anodic carbon steel and cannot be ignored. ${ }^{32-34}$ However, the accelerating effect of the Lorentz forces play a bigger role.

However, the corrosion potential variation does not directly prove the effect of magnetic field on the corrosion behavior of the carbon steel. Therefore, the corrosion current density variation needs to be observed via potentiostatic polarization measurements under different magnetic field conditions to further confirm the effect of magnetic field on the corrosion behavior of carbon steel.

\subsection{Potentiostatic polarization measurement}

Fig. 1 shows that corrosion products will inevitably be produced on the surface of carbon steel after magnetic field pretreatment, and this will affect the further corrosion of carbon steel in seawater. ${ }^{33}$ Fig. 2 shows the corrosion current density variation of three-cases' samples over the time via the potentiostatic polarization measurement without external magnetic field. In all cases, the corrosion current densities $(j)$ gradually decreased over time $(t)$ from the beginning $(0 \mathrm{~s})$. These do not go into a stable state until about $100 \mathrm{~s}$ later. Repeated experiments showed that the corrosion current density linearly decreases 


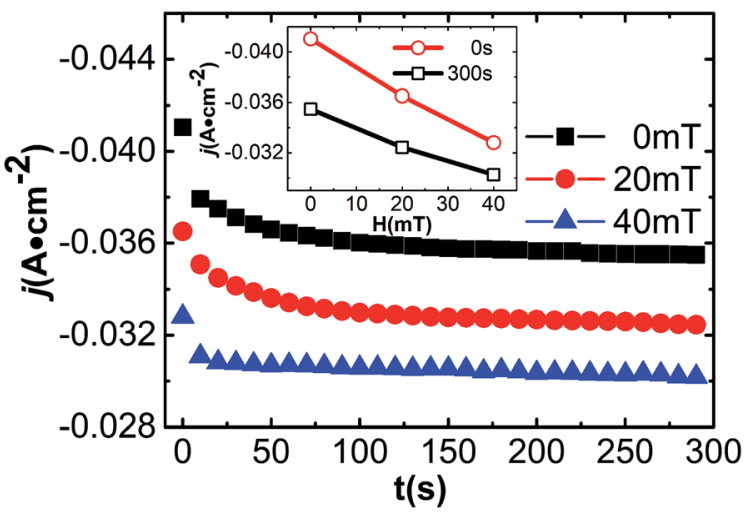

Fig. 2 Potentiostatic polarization curves over time for three-cases samples, the inset shows electrochemical corrosion current densities at $0 \mathrm{~s}$ and $300 \mathrm{~s}$ with changing the strength of the pretreating magnetic field.

with increasing strength of the pretreating magnetic field, and the descending slope of the corrosion current density in the stable state at $300 \mathrm{~s}$ is larger than the initial state at $0 \mathrm{~s}$ (Fig. 2 inset). This result shows that the charge transfer (a secondary mass transfer effect) in the carbon corrosion process should be affected by magnetic field pretreatment. ${ }^{20}$

\subsection{Potentiodynamic polarization measurements}

To determine the effect of magnetic field pretreatment on the electrochemical corrosion behavior of carbon steel in static seawater, potentiodynamic polarization curves were measured for pretreated samples under three cases (Fig. 3). Tafel extrapolation was used to create potentiodynamic polarization curves for pretreated samples under the three cases; these curves were then quantitatively analyzed. By comparing the slopes of the cathode and the anode, we see that the oxidation reaction at the anode (carbon steel) plays a dominant role in the electrochemical corrosion reaction..$^{32}$ With increasing strength of the pretreating magnetic field $(0 \mathrm{mT}$ to $40 \mathrm{mT})$, the electrochemical

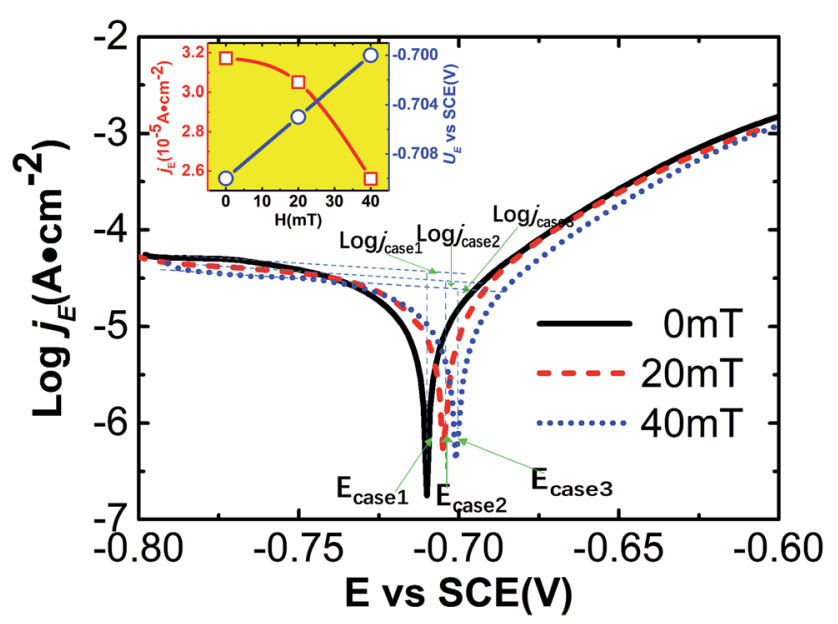

Fig. 3 Potentiodynamic polarization curves for three-cases samples, the inset shows electrochemical corrosion potentials and current densities with changing the strength of the pretreating magnetic field. corrosion potential $\left(U_{\mathrm{E}}\right)$ gradually and linearly approaches the positive value with a shift of about $10 \mathrm{mV}$. The electrochemical corrosion current density $\left(j_{\mathrm{E}}\right)$ gradually decreased by about $0.6 \times 10^{-5} \mathrm{~A} \mathrm{~cm}^{-2}$ as shown in the inset of Fig. 3. These results show that the products of the surface reaction on carbon steel surface in seawater are a major factor in inhibiting corrosion that can be controlled by the magnetic field pretreatment.

\subsection{Electrochemical impedance spectroscopy}

The EIS for the three case samples was measured to confirm the effect of surface reaction products on the corrosion behavior of carbon steel in seawater. Based on an equivalent circuit model, the individual sub-process of carbon steel corrosion can be deduced by analyzing the EIS response data. The capacitive effect results from surface film effects, charge transfer, and mass transfer in the surface corrosion product layer. ${ }^{36}$

Fig. 4 shows the EIS of the samples pretreated with different magnetic field intensities. The evolution of the Nyquist plots shown in Fig. 4(a) indicate a capacitive semicircle (the first time constant) in the high frequency region and an inductive loop (the second time constant) in the low frequency range. These are consistent with two kinds of time constants observed from the Bode plots in Fig. 4(b). The generation of capacitive semicircle is due to charge transfer and double layer capacitance; ${ }^{37}$ the inductive loop emerged as a relaxation process due to the adsorption of $\mathrm{FeOOH}$ - this is an intermediate product on the surface of electrode. ${ }^{38,39}$ Thus, the equivalent circuit $R_{\mathrm{s}}\left(Q_{\mathrm{dl}}(-\right.$ $\left.\left.R_{\mathrm{ct}}\left(R_{\mathrm{L}} L\right)\right)\right)$ was proposed as depicted in the inset of Fig. $4(\mathrm{a}),{ }^{39}$ where $R_{\mathrm{S}}$ is the solution resistance, $R_{\mathrm{ct}}$ is the charge transfer resistance, $Q_{\mathrm{dl}}$ is the constant phase element (CPE), $L$ is the adsorption inductance, and $R_{\mathrm{L}}$ is the adsorption resistance. In addition, the capacitance of the double layer $\left(C_{\mathrm{dl}}\right)$ is calculated from the following formula: $C_{\mathrm{dl}}=1 /\left(2 \pi f_{\max } R_{\mathrm{ct}}\right)$,where $f_{\max }$ is the frequency value when the imaginary component of the impedance is maximum. All of these data are listed in Table 1, respectively. We found that $C_{\mathrm{dl}}$ decreases with increasing pretreating magnetic field strength due to the gradual adsorption of $\mathrm{FeOOH}$. Term $R_{\mathrm{L}}$ can express the adsorption of $\mathrm{FeOOH}-$ it is a physical process under gradient force because $\mathrm{FeOOH}$ is a magnetic particle. The $R_{\mathrm{ct}}$ characterizes a chemical process and increases from $549.1 \Omega \mathrm{cm}^{2}$ to $770.1 \Omega \mathrm{cm}^{2}$ as the magnetic field strength increases (Fig. 4(c)). There have been more obvious changes compared with an increase in $R_{\mathrm{L}}$ from $170 \Omega$ $\mathrm{cm}^{2}$ to $225.1 \Omega \mathrm{cm}^{2}$, which indicates that the thickening of oxide layer on the surface caused by charge transfer plays a greater role than the adsorption. In other words, the charge transfer and adsorption processes can be affected by the magnetic field even if the charge transfer is more obviously affected. Besides, the overall resistance has been found to show an upward trend, which can also explain the reduction in corrosion current density in potentiostatic polarization measurements with increasing pretreatment magnetic field (see Fig. 2). In summary, the effect of the magnetic field on the formation of surface reaction products of carbon steel in seawater can be explained by the MHD effect ${ }^{20-22}$ during the pretreatment process of carbon steel samples. 

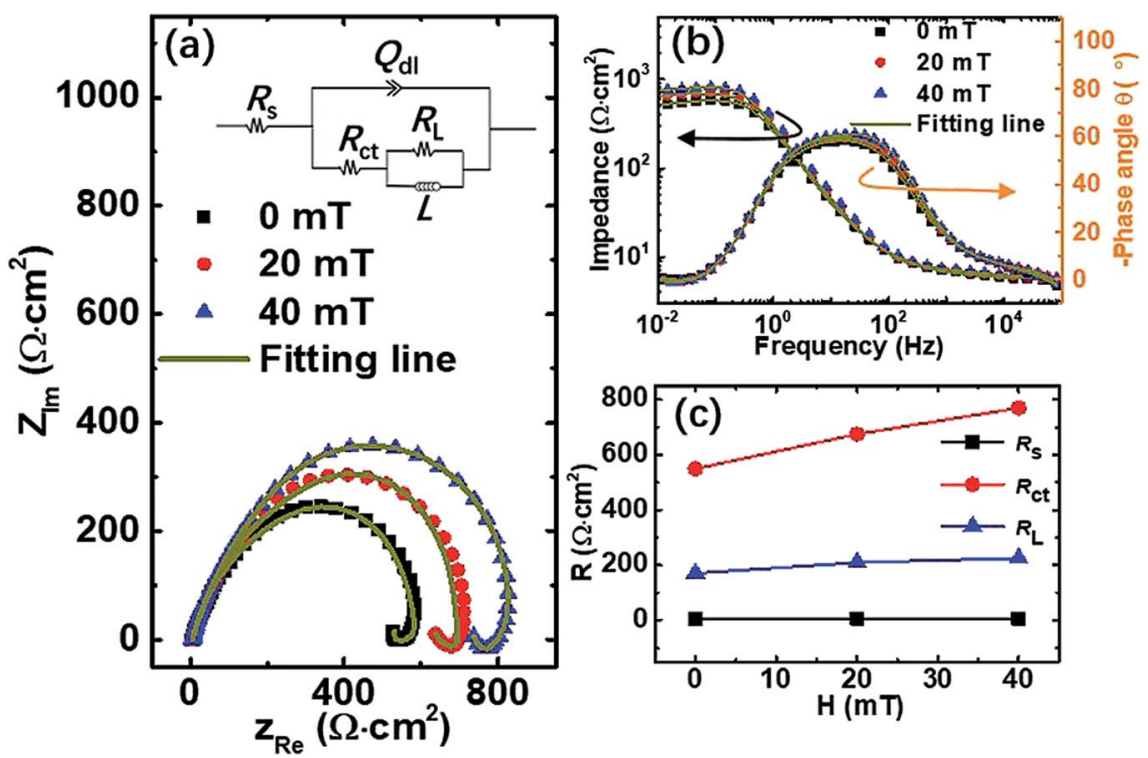

Fig. 4 Evolutions of (a) Nyquist plots and (b) Bode plots for samples pretreated by different magnetic fields. The inset of (a) is equivalent circuit to fit EIS data. (c) Changes of $R_{\mathrm{S}} R_{\mathrm{ct}}$ and $R_{\mathrm{L}}$ with increasing the strength of pretreating magnetic field.

\subsection{Microstructure and composition characterizations}

We next evaluated the effects of surface reaction products on the corrosion behavior of carbon steel in seawater. The microstructure of surface corrosion products in the effective corrosion window of carbon steels after three magnetic field pretreatments was characterized with SEM (Fig. 5(a)). Case-1 (0 mT) has a petallike structure with many holes as shown in the inset of Fig. 5(b). Case-2 $(20 \mathrm{mT})$ has a petal-like structure with many holes and a worm-like structure on the surface of petal-like structures (Fig. 5(c)). Case-3 (40 mT) has a petal-like structure that nearly disappeared. There was only a worm-like structure on the surface of corrosion products (Fig. 5(c)). According to Gu et al., ${ }^{40}$ petallike and the worm-like structures may be an intermediate product of incomplete oxidation in the corrosion process. Furthermore, the compactness of corrosion products on the surface of the carbon steel was significantly enhanced by increasing the pretreated magnetic field strength. This indicates that the magnetic field can affect the formation of surface corrosion products, which agrees with the EIS analysis.

Two kinds of samples were pretreated by magnetic field and naturally dried in air at room temperature or in a $70{ }^{\circ} \mathrm{C}$ oven. These were studied with XRD to determine the composition of the corrosion products on the surface of the carbon steel. Fig. 6(a) shows that the composition of the three cases is almost the same and consists mainly of $\alpha-\mathrm{FeOOH}, \gamma-\mathrm{FeOOH}$, and $\mathrm{NaCl}$ (dried naturally in air at room temperature without any cleaning process). Here, the peaks of $\mathrm{NaCl}$ are come from the seawater, and the peaks of various $\mathrm{FeOOH}$ are from corrosion products of the carbon steel.

To confirm this hypothesis, samples for the three cases were artificially dried by the oven in air at $70{ }^{\circ} \mathrm{C}$ with the cleaning process. Fig. 6(b) shows that the composition of the three cases changed and mainly consists of $\alpha-\mathrm{FeOOH}, \gamma-\mathrm{FeOOH}$, and $\mathrm{Fe}_{2} \mathrm{O}_{3}$; $\mathrm{Fe}_{2} \mathrm{O}_{3}$ is produced after the artificial drying due to the oxidation of iron with oxygen in the air. The results show that the various $\mathrm{FeOOH}$ species must be an intermediate product in the oxidation of iron to $\mathrm{Fe}_{2} \mathrm{O}_{3}$ and confirms that the environment contains $\mathrm{Cl}^{-} ;^{\mathbf{4 1}}$ the specific chemical reaction process is as follows: $:^{\mathbf{4 2 , 4 3}}$

$$
\begin{gathered}
\mathrm{Fe} \rightarrow \mathrm{Fe}^{2+}+2 \mathrm{e} \\
\mathrm{Fe}^{2+} \rightarrow \mathrm{Fe}^{3+}+\mathrm{e} \\
\mathrm{Fe}^{3+}+3 \mathrm{OH}^{-} \rightarrow \mathrm{FeOOH}+\mathrm{H}_{2} \mathrm{O}, \\
\mathrm{Fe}^{2+}+8 \mathrm{FeOOH}+2 \mathrm{e} \rightarrow 3 \mathrm{Fe}_{3} \mathrm{O}_{4}+4 \mathrm{H}_{2} \mathrm{O}, \\
3 \mathrm{Fe}_{3} \mathrm{O}_{4}+1 / 2 \mathrm{O}_{2} \rightarrow 3 \mathrm{Fe}_{2} \mathrm{O}_{3} .
\end{gathered}
$$

\begin{tabular}{|c|c|c|c|c|c|c|c|c|}
\hline $\begin{array}{l}\text { Magnetic field } \\
(\mathrm{mT})\end{array}$ & $R_{\mathrm{S}}\left(\Omega \mathrm{cm}^{2}\right)$ & $Y_{\mathrm{dl}}\left(\mathrm{s}^{n} \Omega^{-1} \mathrm{~cm}^{-2}\right)$ & $n_{\mathrm{dl}}$ & $R_{\text {ct }}\left(\Omega \mathrm{cm}^{2}\right)$ & $R_{\mathrm{L}}\left(\Omega \mathrm{cm}^{2}\right)$ & $L(\mathrm{H})$ & $f_{\max }(\mathrm{Hz})$ & $C_{\mathrm{dl}}\left(\mathrm{F} \mathrm{cm}^{-2}\right)$ \\
\hline 0 & 5.766 & 0.0005376 & 0.7565 & 549.1 & 170 & 249.6 & 0.1 & 0.002898 \\
\hline 40 & 6.131 & 0.0003641 & 0.7808 & 770.1 & 225.1 & 382.4 & 0.08254 & 0.002504 \\
\hline
\end{tabular}

Magnetic field pretreatment can affect the corrosion behavior of carbon steel in static seawater. This is because the magnetic field affects the MHD effect and movement of ions in static

Table 1 Impedance data for samples pretreated by different magnetic fields 

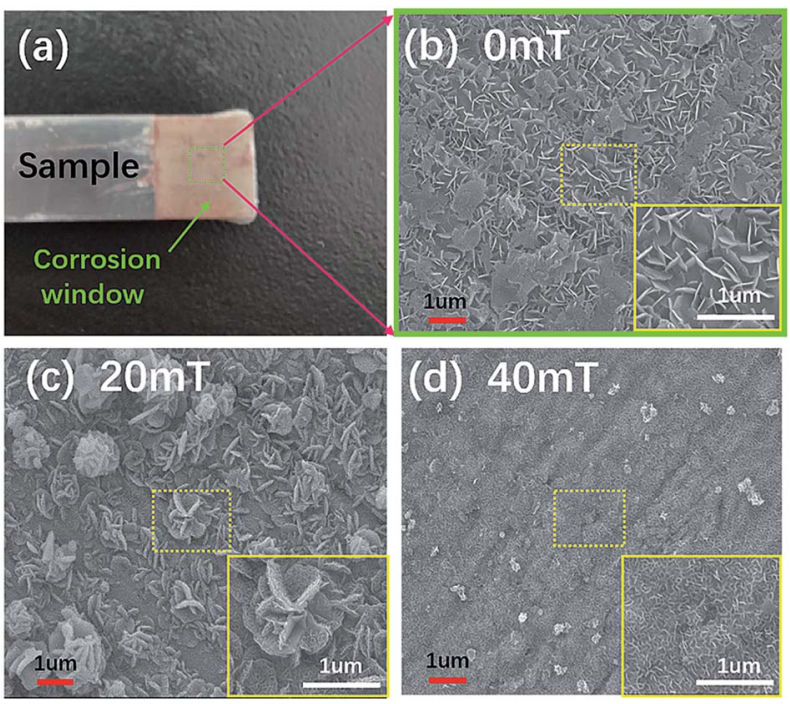

Fig. 5 (a) Microstructure characterizations of corrosion products on the carbon steels surface in seawater for (b) Case-1 (c) Case-2, and (d) Case- 3 samples by SEM. Insets show the zooming in on yellow dotted box areas in figure $(b-d)$.

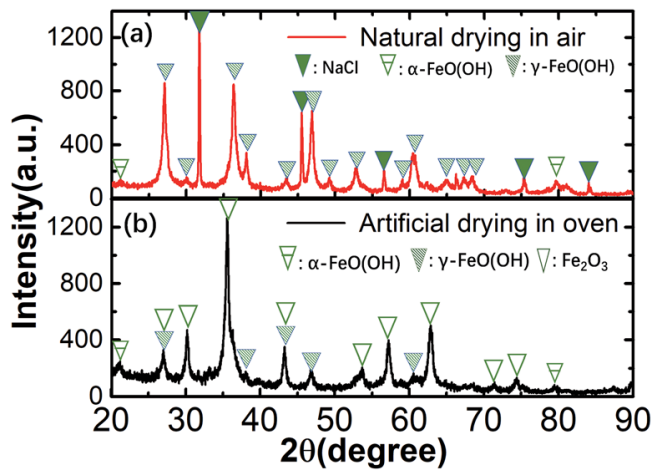

Fig. 6 Composition analysis by XRD for corrosion products by (a) naturally drying in air at room temperature and (b) artificially drying in oven at $70^{\circ} \mathrm{C}$.

seawater. ${ }^{24}$ The Lorentz force will promote the corrosion process of carbon steel via enhanced transfer. ${ }^{20-22}$ Thus, a compact corrosion product can be formed on the carbon steel surface-this helps inhibit the corrosion behavior of carbon steel in seawater.

\section{Conclusions}

We evaluated the effect of magnetic field pretreatment on the corrosion behavior of carbon steel in seawater via electrochemical methods. Although both the charge transfer and adsorption process are affected by the magnetic field, the effect of magnetic field on charge transfer is more obvious. Pretreatment with a magnetic field can effectively inhibit the corrosion behavior of carbon steel by enhancing the compactness of the corrosion products on the carbon steel surface. The magnetic field pretreatment scheme will provide new ways for marine anti-corrosion technology.

\section{Conflicts of interest}

There are no conflicts to declare.

\section{Acknowledgements}

This work was supported by the National Key R\&D Program of China (Grant No. 2017YFB0903700 \& 2017YFB0903702).

\section{References}

1 Y. Li and C. Ning, Latest research progress of marine microbiological corrosion and bio-fouling, and new approaches of marine anti-corrosion and anti-fouling, Bioactive Materials, 2019, 4, 189-195.

2 B. Da, H. Yu, H. Ma and Z. Wu, Reinforcement corrosion research based on the linear polarization resistance method for coral aggregate seawater concrete in a marine environment, Anti-Corros. Methods Mater., 2018, 65, 458-470.

3 P. Cristiani, G. Perboni and A. Debenedetti, Effect of chlorination on the corrosion of $\mathrm{Cu} / \mathrm{Ni} 70 / 30$ condenser tubing, Electrochim. Acta, 2008, 54, 100-107.

4 T. P. Mohan, M. R. Kumar and R. Velmurugan, Mechanical and barrier properties of epoxy polymer filled with nanolayered silicate clay particles, J. Mater. Sci., 2006, 41, 2929-2937.

5 L. Guadagno, L. Vertuccio, A. Sorrentino, M. Raimondo, C. Naddeo, V. Vittoria, G. Iannuzzo, E. Calvi and S. Russo, Mechanical and barrier properties of epoxy resin filled with multi-walled carbon nanotubes, Carbon, 2009, 47, 2419-2430.

6 M. Nematollahi, M. Heidarian, M. Peikari, S. M. Kassiriha, N. Arianpouya and M. Esmaeilpour, Comparison between the effect of nanoglass flake and montmorillonite organoclay on corrosion performance of epoxy coating, Corros. Sci., 2010, 52, 1809-1817.

7 C. A. Loto, R. T. Loto and P. A. Popoola, Evaluation of cathodic protection of mild steel with magnesium anodes in 0.5 M HCL, Chemical Data Collections, 2019, 22, 100246.

8 S. D. Jagtap, S. P. Tambe, R. N. Choudhari and B. P. Mallik, Mechanical and anticorrosive properties of non toxic coal-tar epoxy alternative coating, Prog. Org. Coat., 2014, 77, 395-402.

9 J. Novák, L. Strašák, L. Fojt, I. Slaninová and V. Vetterl, Effects of low-frequency magnetic fields on the viability of yeast Saccharomyces cerevisiae, Bioelectrochemistry, 2007, 70, 115-121.

10 J. Filipič, B. Kraigher, B. Tepuš, V. Kokol and I. MandicMulec, Effects of low-density static magnetic fields on the growth and activities of wastewater bacteria Escherichia coli and Pseudomonas putida, Bioresour. Technol., 2012, 120, 225-232.

11 L. Fojt, L. Strašák, V. Vetterl and J. Šmarda, Comparison of the low-frequency magnetic field effects on bacteria Escherichia coli, Leclercia adecarboxylata and Staphylococcus aureus, Bioelectrochemistry, 2004, 63, 337-341.

12 W. Ji, H. Huang, A. Deng and C. Pan, Effects of static magnetic fields on Escherichia coli, Micron, 2009, 40, 894898. 
13 A. Karantonis, D. Koutsaftis, M. Bredaki and N. Kouloumbi, Reception and detection of chemical signaling by electrochemical oscillators, Chem. Phys. Lett., 2008, 460, 182-186.

14 L. Procópio, The role of biofilms in the corrosion of steel in marine environments, World J. Microbiol. Biotechnol., 2019, 35, 73.

15 Z. P. Lu and J. M. Chen, Magnetic field effects on anodic polarisation behaviour of iron in neutral aqueous solutions, Br. Corros. J., 2000, 35, 224-228.

16 Z. Lu, C. Huang, D. Huang and W. Yang, Effects of a magnetic field on the anodic dissolution, passivation and transpassivation behaviour of iron in weakly alkaline solutions with or without halides, Corros. Sci., 2006, 48, 3049-3077.

17 Y. C. Tang and A. J. Davenport, Magnetic field effects on the corrosion of artificial pit electrodes and pits in thin films, $J$. Electrochem. Soc., 2007, 154, C362.

18 C. Wang, S. Chen, X. Yang and L. Li, Investigation of chloride-induced pitting processes of iron in the $\mathrm{H}_{2} \mathrm{SO}_{4}$ solution by the digital holography, Electrochem. Commun., 2004, 6, 1009-1015.

19 R. Aogaki, T. Negishi, M. Yamato, E. Ito and I. Mogi, Hysteresis effect of magnetic field on electron transfer processes in electrochemical reaction, Physica B, 1994, 201, 611-615.

20 A. Bund and A. Ispas, Influence of a static magnetic field on nickel electrodeposition studied using an electrochemical quartz crystal microbalance, atomic force microscopy and vibrating sample magnetometry, J. Electroanal. Chem., 2005, 575, 221-228.

21 R. Peipmann, J. Thomas and A. Bund, Electrocodeposition of nickel-alumina nanocomposite films under the influence of static magnetic fields, Electrochim. Acta, 2007, 52, 5808-5814.

22 M. Waskaas and Y. I. Kharkats, Effect of magnetic fields on convection in solutions containing paramagnetic ions, $J$. Electroanal. Chem., 2001, 502, 51-57.

23 G. Hinds, J. M. D. Coey and M. E. G. Lyons, Influence of magnetic forces on electrochemical mass transport, Electrochem. Commun., 2011, 3, 215-218.

24 H. Li, Q. Xiong, Z. Lu, J. Chen, Q. Xiao, X. Ru, S. Lin, J. Ma and $\mathrm{Z}$. Chen, A magnetic field induced undulated surface and the shift of the active/passivation transition threshold of iron in a sulfuric acid solution, Corros. Sci., 2017, 129, 179-191.

25 L. M. A. Monzon and J. M. D. Coey, Magnetic fields in electrochemistry: the Lorentz force. a mini-review, Electrochem. Commun., 2014, 42, 38-41.

26 G. Hinds, F. E. Spada, J. M. D. Coey, T. R. Ní. Mhíocháin and M. E. G. Lyons, Magnetic Field Effects on Copper Electrolysis, J. Phys. Chem. B, 2001, 105, 9487-9502.

27 S. Koehler and A. Bund, Investigations on the Kinetics of Electron Transfer Reactions in Magnetic Fields, J. Phys. Chem. B, 2006, 110, 1485-1489.

28 O. Devos, O. Aaboubi, J. P. Chopart, E. Merienne, A. Olivier, C. Gabrielli and B. Tribollet, Eis investigation of zinc electrodeposition in basic media at low mass transfer rates induced by a magnetic field, J. Phys. Chem. B, 1999, 103, 496-501.

29 R. N. O'Brien and K. S. V. Santhanam, Magnetic field assisted convection in an electrolyte of nonuniform magnetic susceptibility, J. Appl. Electrochem., 1997, 27, 573-578.

30 M. Waskaas and Y. I. Kharkats, Magnetoconvection Phenomena: A Mechanism for Influence of Magnetic Fields on Electrochemical Processes, J. Phys. Chem. B, 1999, 103, 4876-4883.

31 Y. Zou, J. Wang, Q. Bai, L. L. Zhang, X. Peng and X. F. Kong, Potential distribution characteristics of mild steel in seawater, Corros. Sci., 2012, 57, 202-208.

32 R. Sueptitz, K. Tschulik, M. Uhlemann, A. Gebert and L. Schultz, Impact of magnetic field gradients on the free corrosion of iron, Electrochim. Acta, 2010, 55, 5200-5203.

33 R. Sueptitz, K. Tschulik, M. Uhlemann, L. Schultz and A. Gebert, Magnetic field effects on the active dissolution of iron, Electrochim. Acta, 2011, 56, 5866-5871.

34 R. Sueptitz, K. Tschulik, M. Uhlemann, J. Eckert and A. Gebert, Retarding the corrosion of iron by inhomogeneous magnetic fields, Mater. Corros., 2014, 65, 803-808.

35 X. Li, M. Zhang, B. Yuan, L. Li and C. Wang, Effects of the magnetic field on the corrosion dissolution of the 304 $\mathrm{SS} \mid \mathrm{FeCl}_{3}$ system, Electrochim. Acta, 2016, 222, 619-626.

36 Y. Xin, T. Hu and P. K. Chu, Degradation behaviour of pure magnesium in simulated body fluids with different concentrations of, Corros. Sci., 2011, 53, 1522-1528.

37 S. M. Bhola, S. Kundu, R. Bhola, B. Mishra and S. Chatterjee, Electrochemical Study of Diffusion Bonded Joints between Micro-Duplex Stainless Steel and $\mathrm{Ti}_{6} \mathrm{~A}_{14} \mathrm{~V}$ Alloy, J. Mater. Sci. Technol., 2014, 30, 163-171.

38 Z. Tao, S. Zhang, W. Li and B. Hou, Corrosion inhibition of mild steel in acidic solution by some oxo-triazole derivatives, Corros. Sci., 2009, 51, 2588-2595.

$39 \mathrm{~W}$. Xu, E. Han and Z. Wang, Effect of tannic acid on corrosion behavior of carbon steel in $\mathrm{NaCl}$ solution, $J$. Mater. Sci. Technol., 2019, 35, 64-75.

40 J. Gu, Y. Xiao, N. Dai, X. Zhang, Q. Ni and J. Zhang, The Suppression of transformation of $\gamma-\mathrm{FeOOH}$ to $\alpha-\mathrm{FeOOH}$ accelerating the steel corrosion in simulated industrial atmospheric environment with a DC electric field interference, Corros. Eng., Sci. Technol., 2019, 54, 249-256.

41 M. Yamashita, H. Konishi, T. Kozakura, J. Mizuki and H. Uchida, In situ observation of initial rust formation process on carbon steel under $\mathrm{Na}_{2} \mathrm{SO}_{4}$ and $\mathrm{NaCl}$ solution films with wet/dry cycles using synchrotron radiation $\mathrm{X}$ rays, Corros. Sci., 2005, 47, 2492-2498.

42 Y. Ma, Y. Li and F. Wang, The effect of $\beta-\mathrm{FeOOH}$ on the corrosion behavior of low carbon steel exposed in tropic marine environment, Mater. Chem. Phys., 2008, 112, 844852.

43 K. Sugae, T. Kamimura, R. Asakura, T. Doi, H. Miyuki and T. Kudo, Electrochemical reduction and re-oxidation behavior of $\alpha, \beta$, and $\gamma$-iron oxy-hydroxide films on electrodes, Mater. Corros., 2019, 70, 187-196. 